\title{
Psychometric Properties of the Chinese Version of the Pittsburgh Sleep Quality Index (PSQI) Among Hong Kong Chinese Childhood Cancer Survivors
}

Ka Yan HO ( $\sim$ kyeva.ho@polyu.edu.hk )

The Hong Kong Polytechnic University https://orcid.org/0000-0003-3953-9065

Katherine K.W. Lam

The Hong Kong Polytechnic University

\section{Xia W}

Sun Yan-sen University of Medical Sciences: Sun Yat-Sen University

\section{J.O.K. Chung}

The Hong Kong Polytechnic University

\section{Ankie T. Cheung}

University of Hong Kong

\section{Laurie L.K. Ho}

The Hong Kong Polytechnic University

\section{S. Y. Chiu}

Hong Kong Children's Hospital

\section{G.C.F. Chan}

Hong Kong Children's Hospital

William H.C. Li

University of Hong Kong

\section{Research}

Keywords: Sleep disruption, Childhood cancer survivors, Assessment, Psychometric properties

Posted Date: April 20th, 2021

DOl: https://doi.org/10.21203/rs.3.rs-435552/v1

License: (c) (i) This work is licensed under a Creative Commons Attribution 4.0 International License. Read Full License July 6th, 2021. See the published version at https://doi.org/10.1186/s12955-021-01803-y. 


\section{Abstract}

\section{Background}

Sleep disruption is a prevalent symptoms reported by survivors of childhood cancer. However, there is no validated instrument for assessing this symptom in this population group. To bridge the literature gap, this study translated and adapted the Pittsburgh Sleep Quality Index (PSQI) for Hong Kong Chinese cancer survivors and examined its psychometric properties and factor structure.

\section{Methods}

A convenience sample of 402 Hong Kong Chinese childhood cancer survivors aged 6-18 years were asked to complete the Chinese version of the PSQI, Center for Epidemiologic Studies Depression Scale for Children (CES-DC), Fatigue Scale-Child (FS-C)/Fatigue Scale-Adolescent (FS-A), and Pediatric Quality of Life Inventory (PedsQL). To assess known-group validity, 50 pediatric cancer patients and 50 healthy counterparts were recruited. A sample of 40 children were invited to respond by phone to the PSQI 2 weeks later to assess test-retest reliability. A cutoff score for the translated PSQI used with the survivors was determined using receiver operating characteristic analysis.

\section{Results}

The Chinese version of the PSQI had a Cronbach alpha of 0.71, with an intraclass correlation coefficient of 0.90. Childhood cancer survivors showed significantly lower mean PSQI scores than children with cancer, and significantly higher mean scores than healthy counterparts. We observed positive correlations between PSQI and CES-DC scores and between PSQI and FS-A/FS-C scores, but a negative correlation between PSQI and PedsQL scores. Confirmatory factor analysis showed that the translated PSQI data best fit a three-factor model. The best cutoff score to detect insomnia was 5 , with a sensitivity of 0.81 and specificity of 0.70 .

\section{Conclusion}

The Chinese version of the PSQI is a reliable and valid instrument to assess subjective sleep quality among Hong Kong Chinese childhood cancer survivors. The validated PSQI could be used in clinical settings to provide early assessments for sleep disruption. Appropriate interventions can therefore be provided to minimize its associated long-term healthcare cost.

\section{Trial registration}

This study was registered in ClinicalTrials.gov with the reference number NCT03858218.

\section{Background}


Cancer is a major cause of death in the pediatric population. In Hong Kong, 46 patients aged 0-19 years died from cancer in 2016 [1]. According to the Hong Kong Cancer Registry, approximately 190 new cases of childhood cancer are diagnosed each year [2]. Although this figure is lower than for adults, childhood cancer remains a significant healthcare concern because of its damaging effects on children's physical and psychological functions throughout life [3]. Particularly, children surviving cancer still have to bear the health burden of numerous sequelae, even if their treatment finished months or even years previously [4].

One of the most prevalent symptoms reported by patients who survive cancer is sleep disruption [5]. It is defined as a range of difficulties in achieving enough good quality sleep [6]. One systematic review reported that $25-59 \%$ of adults report sleep disruption after cancer treatment [5]. A retrospective cohort study found that $16.7 \%$ of adult survivors of childhood cancer complained of disrupted sleep [7]. The causes of sleep disruption are multifactorial [5]. However, it can largely attributed to disruption of circadian rhythm by prolonged use of medications and long-term hospitalization [5]. Sleep disruption can have severe negative effects on survivors of cancer [8]; it precipitates inflammation and oxidative stress, which contribute to neurocognitive impairment [9]. Sleep disruption can also lead to fatigue [10], which limits patients' capacity to engage in daily activities [11] and results in depression [12], severely compromising patients' long-term quality of life [13]. Therefore, healthcare professionals should recognize their responsibility to implement appropriate interventions to improve cancer survivors' sleep quality and minimize the associated health consequences of sleep disruption. The development and evaluation of such interventions requires a reliable and valid instrument that can precisely assess the sleep quality of cancer survivors.

The Pittsburgh Sleep Quality Index (PSQI) is the most commonly used instrument to assess the subjective sleep quality of adults in clinical and community settings [14]. Although this instrument was originally developed for psychiatric patients [15], it has been validated in different populations of adult cancer patients $[16,17]$ and extensively applied in studies of this population [14]. Increasingly, the PSQI has been used to assess children's sleep quality [18]; there is evidence that it is a reliable and valid instrument for young people [19], community-based adolescents [20], and college students [21]. However, the PSQI has never been validated for use with pediatric patients, including childhood cancer survivors [22]. As cancer may affect survivors' experience of sleep disruption and symptom presentation, direct application of the PSQI to assess their sleep quality may therefore lead to inaccurate results. One literature review indicated that no validated instrument is available for assessing sleep quality in childhood cancer survivors [22]. This lack of validated instruments precludes understanding the severity of the problem and hinders the development of appropriate interventions to promote sleep quality.

\section{Methods}

Aim 
This study aimed to translate and adapt the Pittsburgh Sleep Quality Index (PSQI) for Hong Kong Chinese cancer survivors and examine its psychometric properties and factor structure.

\section{Design}

A cross-sectional study was conducted to validate the Chinese version of the PSQI. The study was carried out in an outpatient clinic of a public acute care hospital in Hong Kong.

\section{Sample/ Participants}

Hong Kong Chinese childhood cancer survivors who had outpatient medical follow-ups were invited. Eligible survivors were aged 6-18 years, and able to communicate in Cantonese and read Chinese. We excluded survivors whose medical records identified cognitive or behavioral problems. To ensure that all participants could understand the questionnaire, we included only those older than 6 years.

To assess the known-group validity, 50 pediatric cancer patients and 50 healthy counterparts were recruited using the same inclusion and exclusion criteria.

There is no universal guideline on the minimal sample size required for confirmatory factor analysis. Nunnally [23] suggested that at least 10 subjects are required for each item. As the PSQI contains 19 items, we planned to recruit at least 380 childhood cancer survivors.

\section{Instrument}

\section{(1) Demographic and clinical characteristics}

A structured questionnaire was used to record participant demographic and clinical characteristics.

(2) The Chinese version of the Pittsburgh Sleep Quality Index (PSQI)

The 19-item PSQI assesses subjective sleep quality in the last month. Items are categorized into seven components: subjective sleep quality, sleep latency, sleep duration, habitual sleep efficiency, sleep disturbances, sleep medication, and daytime dysfunction. The possible score range for each component is 0 (no difficulty) to 3 (severe difficulty). The seven component scores are summed to produce a global score; higher scores represent poorer subjective sleep quality. Previous psychometric studies of the English version of the PSQI have confirmed that it is a reliable and valid instrument for assessing subjective sleep quality among adult cancer survivors [24]. The factor structure of the PSQI has also been widely examined $[15,25,26]$. Results vary according to sample characteristics $[15,25,26]$. Although Buysse et al.[15] suggested a single-factor model underlying the PSQI in depressive patients, Cole et al. [25] proposed a three-factor model in older adults and Magee et al. [26] identified a two-factor model in Australian adults.

(3) The Chinese version of the Center for Epidemiologic Studies Depression Scale for Children (CES-DC) 
The CES-DC assesses depressive symptoms in people aged 6-17 years. It comprises 20 items measured on a 4-point scale. Higher scores represent more depressive symptoms. This is a reliable and valid instrument for children [27].

(4) The Chinese version of the Fatigue Scale-Child (FS-C)/Fatigue Scale-Adolescent (FS-A)

These two scales measure cancer-related fatigue levels in pediatric oncology patients. The FS-C comprises 13 items for patients aged 7-12 years; the FS-A contains 12 items for patients aged 13-18 years. Higher scores represent higher levels of cancer-related fatigue. They are reliable and valid measures of cancer-related fatigue among Hong Kong Chinese childhood cancer survivors $[28,29]$.

(5) The Chinese version of the Pediatric Quality of Life Inventory 4.0 Generic Core Scale (PedsQL 4.0)

The PedsQL assesses health-related quality of life of people aged 2-18 years. This scale comprises 23 items categorized into four different subscales: physical functioning, emotional functioning, social functioning, and school functioning. Subscale scores are obtained by averaging item scores for the corresponding subscales; the total scale score is the average of all item scores. Higher PedsQL scores indicate better quality of life. The psychometric properties of this scale among Chinese children have been well established [30].

\section{Study Procedures}

An expert panel was set up for translation. The panel contained an associate professor, two assistant professors, a research assistant professor, a postdoctoral fellow, a pediatric oncologist, and a nurse specialist. The procedures suggested by Bracken and Barona [31] were followed. The PSQI was translated from English into Chinese by two independent bilingual translators. The two translations were compared and reconciled after panel member discussions. Another two independent bilingual translators blinded to the original English version of the scale were asked to translate the Chinese version back into English. The panel members compared the back translations against the original English version and decided whether the meaning of each item in the back translations had been retained. Disagreement was resolved during regular meetings.

After the informed consent process, parents were invited to complete a simple questionnaire that documented their child's demographic and clinical characteristics. Children were asked to complete the PSQI, CES-DC, FS-A/FS-C, and PedsQL. To assess test-retest reliability, a sample of 40 children were invited to respond by phone to the PSQI 2 weeks later.

\section{Statistical Analysis}

For sematic equivalence, the expert panel were asked to compare the original and translated versions of the PSQI, and rate the equivalence of each translated item using a 4-point scale (from $1=$ not equivalent to $4=$ most equivalent). The equivalence rate was calculated as the percentage of items rated as either 3 or 4 . Any item rated 1 or 2 by $20 \%$ of the experts was deemed not equivalent and was amended. 
For content equivalence, the expert panel rated the relevancy of each PSQI item to the concept (i.e., subjective sleep quality) using a 4 -point scale (from $1=$ not relevant to $4=$ very relevant). The content validity index $(\mathrm{CVI})$ of an item (I-CVI) was calculated as the percentage of experts who rated the item as either 3 or 4 . A CVI of the translated PSQI (S-CVI) was the average of the I-CVIs for all items. An I-CVI $\geq$ 0.78 or higher and $\mathrm{S}-\mathrm{CVI} \geq 0.9$ were considered acceptable [32].

The internal consistency of the Chinese version of the PSQI was evaluated using Cronbach's alpha, and the test-retest reliability using the intraclass correlation coefficient (ICC).

Convergent validity was established by identifying correlations between PSQI and CES-DC scores, between PSQI and FS-A/FS-C scores, and between PSQI and PedsQL scores. Following previous studies $[10,12,13]$, we hypothesized positive correlations between PSQI and CES-DC scores and between PSQI and FS-A/FS-C scores, but a negative correlation between PSQI and PedsQL scores.

Confirmatory factor analysis was performed. The overall fits of the single-factor, two-factor, and threefactor models were examined and compared because previous studies report varying PSQI factor structures $[15,25,26]$. The overall model fit was determined using following fit indices: the $\chi^{2} /$ degrees of freedom $(d f)$ ratio, root mean square error of approximation (RMSEA), and comparative fix index (CFI).

A cutoff score for the translated PSQI used with the survivors was determined using receiver operating characteristic (ROC) analysis. Participants were regarded as having insomnia if they met all diagnostic criteria for insomnia in Diagnostic and Statistic Manual of Mental Disorders Fifth Edition (DSM-5). The area under the curve (AUC) was calculated. Sensitivity and specificity were used to determine the best cutoff score.

\section{Ethical considerations}

Ethical approval was obtained from the Institutional Review Board of the University of Hong Kong/Hospital Authority Hong Kong West Cluster (UW 18-366). A research assistant approached all parents in the outpatient clinic, and introduced the study. After checking eligibility, the research assistant obtained written consent from parents who allowed their child to participate. These parents were ensured that withdrawal would not result in any prejudice to the care received. The children were also invited to write their names on a child assent form to indicate their willingness to participate.

\section{Results}

\section{Demographics}

Table 1 shows participant demographics. The mean age of the survivors was 12.3 years (standard deviation $=3.8)$. Of patients, $55.7 \%(n=224)$ were boys and $77.6 \%(n=312)$ of children's parents had upper secondary education or above. Of patients, $66.9 \%(n=269)$ were diagnosed with non-solid tumor, $58.2 \%(n=234)$ had received only one type of treatment, and $57.3 \%(n=230)$ completed their treatment 
less than 5 years ago. Comparative statistics indicated that the three groups were similar in age, sex distribution, and parental educational attainment. 
Table 1

Demographic characteristics of the subjects $(N=502)$

$$
n(\%)
$$

Childhood cancer

Sex

Male

Female

Parents' Educational

Attainment

Lower secondary school $\quad 90(22.4)$

or below

Upper secondary school $312(77.6)$

or above

Diagnosis

Non-solid tumor

Solid tumor

Treatment received

One type of treatment

More than one type of treatment

Age

6-12 years

$13-18$ years

Time since treatment

completed

Less than 5 year

5 to 10 years

$204(50.7)$

198 (49.3)

Mean (SD)

$12.3(3.78)$ survivors

$n=402$

$224(55.7)$

178 (44.3)

Healthy children $x^{2} / F$

$n=50 \quad n=50$

value
$26(52.0)$

24 (48.0)

4.24

$23(46.0)$

27 (54.0)

$1.82 \quad 0.40$

0.12

$15(30.0)$

$17(34.0)$

35 (70.0)

$33(66.0)$
$1.70 * \quad 0.75^{\star}$

$32(64.0)$

$18(36.0)$

133 (33.1)

0.70 * $0.45^{\star}$

$26(52.0)$

24 (748.0)

$168(41.8)$

$1.10 \quad 0.58$

27 (54.0)

$22(44.0)$

$23(46.0)$

$28(56.0)$

$11.8(3.47)$

$12.2(3.59)$

0.63 


\section{$n(\%)$}

More than 10 years

$48(11.9)$

*Significant at $p<0.05$

Validity

\section{Semantic equivalence}

The semantic equivalence ranged from $85.7-100 \%$. The overall rate was $99.2 \%$, indicating that all items of the Chinese version of the PSQI were conceptually and idiomatically equivalent to those in the English version.

\section{Content equivalence}

Regarding the I-CVIs, the ratings ranged from $14.3-100 \%$, with an S-CVI of $95.2 \%$, indicating that most items, except item 8 , reflected the underlying construct. Item 8 was then revised. The recalculated S-CVI and I-CVI were $100 \%$, confirming the content validity.

\section{Construct validity}

Table 2 shows the results of one-way between-subjects analysis of variance and post-hoc testing on PSQI scores for the three groups. Survivors of childhood cancer reported a significantly lower mean PSQI score than children with cancer ( 4.64 vs $6.60, p<0.05$ ), but a significantly higher mean score than their healthy counterparts (4.64 vs $3.38, p<0.05)$. This confirmed the known-group validity.

Table 2

The test results of ANOVA on the levels of fatigue among the three groups

\begin{tabular}{|c|c|c|c|c|c|c|c|c|c|}
\hline & \multicolumn{3}{|c|}{ Mean (SD) } & \multicolumn{2}{|l|}{ G1 vs G2 } & \multicolumn{2}{|l|}{ G1 vs G3 } & \multicolumn{2}{|l|}{ G2 vs G3 } \\
\hline & G1 & $\mathrm{G} 2$ & G3 & $\begin{array}{l}\text { Mean } \\
\text { Difference }\end{array}$ & $\begin{array}{l}p- \\
\text { value }\end{array}$ & $\begin{array}{l}\text { Mean } \\
\text { Difference }\end{array}$ & $\begin{array}{l}p \text { - } \\
\text { value }\end{array}$ & $\begin{array}{l}\text { Mean } \\
\text { Difference }\end{array}$ & $\begin{array}{l}p- \\
\text { value }\end{array}$ \\
\hline $\begin{array}{l}\text { Sleep } \\
\text { quality }\end{array}$ & 4.64 & 6.60 & 3.38 & -1.96 & $0.00 *$ & -1.26 & $0.00 *$ & 3.22 & $0.00 *$ \\
\hline \multicolumn{10}{|c|}{ *Significant at $p<0.05$} \\
\hline
\end{tabular}

Interrelationships between PSQI, CES-DC, FS-C/FS-A, and PedsQL scores among survivors were examined using the Pearson correlation coefficient. Correlation coefficients of $0.10-0.29,0.30-0.49$, and $0.50-1.0$ 
can be interpreted as small, medium, and large, respectively [33]. For survivors aged 6-12 years (Table 3), there was a large positive correlation between PSQI and FS-C scores ( $r=0.60, n=204, p<0.01)$, and between PSQI and CES-DC scores $(r=0.57, n=204, p<0.01)$, and a medium negative correlation between PSQI and PedsQL scores $(r=-0.43, n=204, p<0.01)$. Survivors aged $13-18$ years (Table 3 ) showed a strong positive correlation between PSQI and CES-DC scores $(r=0.64, n=198, p<0.01)$, a small positive correlation between PSQI and FS-A scores $(r=0.27, n=198, p<0.01)$, and a medium negative correlation between PSQI and PedsQL scores $(r=-0.45, n=198, p<0.01)$. This indicated that survivors of childhood cancer who reported more sleep disturbance had more depressive symptoms and cancer-related fatigue and lower quality of life. Construct validity was demonstrated.

Table 3

Interrelationships between the scores of PSQI, CES-DC, FS-C and PedsQL among the survivors aged 6-12 years $(n=204)$ and survivors aged $13-18$ years $(n=198)$.

\section{Survivors aged $6-12$ years $(n=204)$}

\begin{tabular}{|c|c|c|c|c|}
\hline & Global PSQI & CES-DC & FS-C & PedsQL \\
\hline Global PSQI & - & $0.57 \star \star$ & $0.60 * *$ & $-0.43^{\star \star *}$ \\
\hline CES-DC & $0.57 \star \star$ & - & $0.55^{\star \star}$ & -0.32 ** \\
\hline FS-C & $0.60 * \star$ & $0.55^{\star \star}$ & - & $-0.31^{\star \star}$ \\
\hline PedsQL & $-0.43^{\star \star}$ & -0.32 *ᄎ & -0.31 ** & - \\
\hline \multicolumn{5}{|c|}{ Survivors aged $13-18$ years $(n=198)$} \\
\hline & Global PSQI & CES-DC & FS-A & PedsQL \\
\hline Global PSQI & - & $0.64 * \star$ & $0.27 \star \star$ & $-0.45^{\star \star}$ \\
\hline CES-DC & $0.64 \star \star$ & - & $0.32^{\star \star}$ & $-0.57 * \star$ \\
\hline FS-A & $0.27 \star \star$ & $0.32^{\star \star}$ & - & $-0.37 * \star$ \\
\hline PedsQL & $-0.45^{\star \star}$ & $-0.57 * \star$ & $-0.37 \star \star$ & - \\
\hline \multicolumn{5}{|c|}{$\begin{array}{l}\text { Abbreviations: CES-DC, Center for Epidemiologic Studies Depression Scale for Children; FS-A, Fatigue } \\
\text { Scale-Adolescent; FS-C, Fatigue Scale-Child; PedsQL, Pediatric Quality of Life Inventory; PSQI, } \\
\text { Pittsburgh Sleep Quality Index. }\end{array}$} \\
\hline \multicolumn{2}{|c|}{$\star \star=p$-value $<0.01$} & & & \\
\hline
\end{tabular}

\section{Confirmatory factor analysis}

Table 4 presents the fit indices of the Chinese version of the PSQI based on the single-factor, two-factor, and three-factor models. The results indicated that the seven component scores best fit the three-factor model. Figure 1 shows the estimated parameters of the Chinese version of the PSQI based on the threefactor model. All correlation matrices were positive and less than 1, thus were reasonable. Additionally, 
the factor loadings were high, ranging from 0.48 to 0.85 . The $t$-values were greater than 2 and statistically significant. The standard errors were between 0.15 and 0.46 , suggesting that all parameters were accurately estimated.

Table 4

Fit statistics for the Chinese version of the PSQI

\begin{tabular}{|llll|}
\hline Factor model & $x^{2} / \mathbf{d f}$ & CFI & RMSEA \\
\hline 1-factor model & 10.36 & 0.62 & 0.15 \\
\hline 2-factor model & 7.21 & 0.78 & 0.12 \\
\hline 3-factor model & 3.12 & 0.07 & 0.09 \\
\hline $\begin{array}{l}\text { Abbreviations: } x^{2} / \mathrm{df}, \text { Relative chi-square; } \mathrm{CFI} \text {, Comparative fix index; RMSEA, Root Mean Square Error } \\
\text { of Approximation }\end{array}$ & & \\
\hline
\end{tabular}

Footnotes

Confirmatory factor analysis (CFA) was carried out using AMOS version 25.0 for Windows. Acceptable overall fit of each model was evaluated using the following indices:

\begin{tabular}{|ll|}
\hline Criterion & Range \\
\hline$x^{2} / \mathrm{df}$ & $1.00-5.00$ \\
\hline $\mathrm{CFI}$ & 0.9 or higher \\
\hline RMSEA & 0.08 or less \\
\hline
\end{tabular}

\section{Reliability}

The ICC at the 2-week interval was $0.90(p<0.001)$, which is higher than the cutoff of 0.7 , indicating that the Chinese version of the PSQI is acceptable for research. The seven component scores of the translated PSQI achieved a Cronbach alpha of 0.71 . The corrected item-total correlations ranged from 0.27 to 0.63 , indicating acceptable internal consistency.

\section{ROC analysis}

When compared with the DSM-5 diagnostic criteria for insomnia, the Chinese version of the PSQI demonstrated acceptable discrimination, with an AUC of 0.83 (Fig. 2). Table 5 shows the sensitivity and specificity at different cutoff scores. The best cutoff score to detect insomnia was 4.5 , with a sensitivity of 0.81 and specificity of 0.70 . As the global score of the PSQI must be an integer, a score of 5 was chosen as the best cutoff. 
Table 5

Various cutoff scores for the Chinese version of the PSQI

\begin{tabular}{|lll|}
\hline Cutoff score & Sensitivity & Specificity \\
\hline 0.5 & 1.00 & 0.03 \\
1.5 & 1.00 & 0.11 \\
2.5 & 1.00 & 0.28 \\
\hline 3.5 & 0.92 & 0.49 \\
\hline 4.5 & 0.81 & 0.70 \\
\hline 5.5 & 0.66 & 0.82 \\
\hline 6.5 & 0.53 & 0.91 \\
\hline 7.5 & 0.32 & 0.94 \\
\hline 8.5 & 0.15 & 0.97 \\
\hline 9.5 & 0.10 & 0.98 \\
\hline 10.5 & 0.09 & 0.99 \\
\hline 13.0 & 0.03 & 1.00 \\
\hline 16.0 & 0.00 & 1.00 \\
\hline
\end{tabular}

\section{Discussion}

Although sleep disruption is prevalent in pediatric patients after completion of cancer treatment, no validated instrument is available to assess this symptom. To address this lack, we translated and adapted the PSQI, which is commonly used among adult cancer patients, for Hong Kong Chinese cancer survivors. We also examined the psychometric properties of the Chinese version of the PSQI in this population.

Consistent with previous studies of young people and community-based adolescents $[19,20]$, the Chinese version of the PSQI demonstrated acceptable internal consistency; corrected item-total correlations ranged from 0.27 to 0.63 . This indicated that all items in the translated PSQI measure the same construct: sleep quality. Additionally, the ICC at 2 weeks was 0.90 . This is in accordance with previous studies showing that the PSQI had good stability in measuring subjective sleep quality $[19,20]$.

We examined the construct validity of the Chinese version of the PSQI using the known-group technique. In line with some previous studies [6,34], our results indicated that survivors of childhood cancer had a higher mean PSQI score than their healthy counterparts, but a lower mean score than those undergoing 
cancer treatment. This confirmed that the translated PSQI was able to differentiate the sleep quality of different groups of children.

It is well-documented that sleep is one of the most effective ways for the body to restore energy [35]. Inadequate sleep therefore leads to fatigue and affects daily activities $[10,11]$, resulting in depression and lower quality of life $[12,13]$. Consistent with existing literature, we found a negative correlation between PSQI and PedsQL scores. Positive correlations were observed between PSQI and CES-DC scores, and between PSQI and FS-C/FS-A scores, indicating that the Chinese version of the PSQI showed convergent validity.

There is no consensus on the dimensionality of the PSQI [36]. Buysse et al. [15] suggested that the seven components of the PSQI should be combined into a single factor. Cole et al. [25] identified a three-factor model comprising sleep efficiency, sleep quality, and daily dysfunction. Magee et al. [26] proposed a twofactor model because they found an extraordinarily high correlation between the factors of sleep quality and daily disturbance, suggesting that these two factors overlap. The present findings are consistent with those of Cole et al. [25] in that the three-factor model achieved a better fit than the single- and two-factor models. We also observed a reasonable correlation between the factors of sleep quality and daily disturbance, thus confirming that they are two different constructs. All these findings provide empirical evidence that our translated PSQI can assess the sleep quality of survivors of childhood cancer in terms of three separate domains. Merz and Tomfohr-Madsen [6] considered sleep problems in childhood cancer survivors as multidimensional, and conceptualized them as a range of sleeping difficulties related to biological and psychosocial aspects. Our translated PSQI could be used to detect sleep problems located on only one of the three factors. Appropriate interventions could therefore be chosen according to the type and nature of the sleep problem.

The confirmatory factor analysis showed that most components achieved high factor loadings on the three-factor model, except component 6 (the use of sleep medication obtained a factor loading of 0.48 ). This result is similar to findings from other PSQI validation studies in the Chinese population [37]. A possible explanation is that Chinese people do not commonly use sleep medication because they often believe that it has many side effects [38]. Instead, they tend to use complementary and alternative approaches (e.g., acupuncture and aromatherapy) to relieve sleep problems $[39,40]$. This explanation is supported by our findings: $97 \%$ of subjects answered "not during the past month" when responding to item 7 on the PSQI: "how often have you taken medicine (prescribed or over-the-counter) to help you sleep?" In response to this issue, we reran the confirmatory factor analysis by removing this component score from our models. However, this did not greatly improve the fit indices. Future studies should consider examining the role of this component in assessing sleep quality in other pediatric patients.

The confirmatory factor analysis also showed that the use of sleep medication was accounted for by the latent variable of sleep efficiency. This is different from the three-factor model proposed by Cole et al. [25], in which the use of sleep medication was accounted for by the latent variable of sleep quality. Such inconsistency may be because people with sleep problems may take medications for various reasons, 
such as trouble falling asleep (associated with sleep quality) and short sleep duration (related to sleep efficiency). Hence, the use of medications is a poor indicator of latent variables [41].

The ROC analysis results revealed that a global score of $\geq 5$ was the best cutoff score for distinguishing survivors of childhood cancer with and without insomnia under the diagnostic criteria of DSM-5. The AUC was 0.83 , which indicated that the Chinese version of the PSQI has acceptable discrimination. Although our identified cutoff score was slightly lower than that in university students [42], different PSQI cutoff scores have been reported in different studies, according to sample characteristics $[15,43-46]$.

We changed the wording of item 8 with reference to its CVI. In the original version, item 8 asks an individual to report how often he or she had trouble staying awake under different scenarios, including driving. However, this scenario was not relevant to our target population because they were not able to drive (the legal driving age in Hong Kong is 18 years). Schools play an important role in child and youth development [47]. Therefore, our expert panel changed "driving" to "doing homework" to make item 8 more appropriate for our population.

One of the main strengths is the originality of the research question. Our study bridged an existing research gap by translating the PSQI from English into Chinese and examining the psychometrics of the translated scale among childhood cancer survivors. Another strength is that our study was conducted in Hong Kong Children's Hospital, the only hospital in Hong Kong that provides medical consultation for pediatric oncology patients. Hence, the sample was representative.

\section{Limitations}

A major limitation is that the limited budget precluded use of objective sleep measures, such as actigraphy, to validate PSQI results. Another limitation is that although polysomnography (PSG) is regarded as the gold standard to diagnose insomnia, we only used DSM-5 as the diagnostic tool for screening participants with insomnia since the use of PSG is not a routine clinical procedure and is highly complicated with laboratory analysis.

\section{Conclusion}

There is increasing evidence of the serious effects of sleep disruption on survivors of childhood cancer [9-12]. However, the problem is not routinely assessed, which indicates room for improvement in survivorship care [48]. The validated PSQI could be used in clinical settings to monitor the subjective sleep quality of survivors of childhood cancer. This would help to provide early assessments and interventions to alleviate sleep disruption and minimize its associated long-term healthcare cost. Previous systematic reviews have identified different effective interventions to minimize sleep disruption among adult cancer patients [49-50]. However, owing to a lack of validated instruments to assess sleep quality among childhood cancer survivors, these interventions have never been tested in this populations. It thus remains unclear whether they are useful for survivors of childhood cancer. The validated PSQI could be 
used to evaluate the effectiveness of these interventions to reduce sleep disruption among childhood cancer survivors.

This study examined the psychometric properties of the Chinese version of the PSQI. The translated PSQI was demonstrated to be a reliable and valid instrument to assess subjective sleep quality. The threefactor structure of the PSQI proposed by previous studies was confirmed.

\section{Abbreviations}

PSQI: Pittsburgh Sleep Quality Index; CES-DC: Center for Epidemiologic Studies Depression Scale for Children; FS-C: Fatigue Scale-Child; FS-A: Fatigue Scale-Adolescent; PedsQL 4.0: Pediatric Quality of Life Inventory 4.0 Generic Core Scale; CVl: content validity index; I-CVI: item content validity index; S-CVI: scale content validity index; $d f$ : degrees of freedom; RMSEA: root mean square error of approximation; CFI: comparative fix index; ROC: receiver operating characteristic; DSM-5: Diagnostic and Statistic Manual of Mental Disorders Fifth Edition; AUC: area under the curve; PSG: polysomnography

\section{Declarations}

\section{Ethics approval and consent to participate}

This study has been approved by the Institutional Review Board of the University of Hong Kong/Hospital Authority Hong Kong West Cluster (UW 18-366). Written parental consent was obtained after fully informing the study's purpose and details.

\section{Consent for publication}

Not applicable.

\section{Availability of data and materials}

The data will be available upon reasonable request.

\section{Competing interests}

The authors declare that they have no competing interests.

\section{Funding}

This study was supported by Seed Fund for Basic Research for New Staff (Ref: 201805159003), the University of Hong Kong, HKSAR.

\section{Authors' contributions}


All authors contributed in study design. $\mathrm{KYH}, \mathrm{KKWL}$, and LWHC were responsible for data acquisition, analysis, drafting and revising of the manuscript. All authors participated in reading and approving the final manuscript.

\section{Acknowledgements}

We thank the children and parents who had spent their time in our study. We also thank Diane Williams, PhD, from Edanz Group (https://en-author-services.edanz.com/ac) for editing a draft of this manuscript.

\section{References}

1. Children's Cancer Foundation. Childhood Cancer Facts and Figures. https://www.ccf.org.hk/information.php?id=310. Accessed April 12, 2021.

2. Hong Kong Cancer Registry. Hong Kong Cancer Registry 2015. http://www3.ha.org.hk/cancereg/Statistics.html\#sqs. Accessed April 12, 2021.

3. Berk L. Development through the lifespan. India: Pearson Education; 2017.

4. Warner EL, Kirchhoff AC, Nam GE, Fluchel M. Financial Burden of Pediatric Cancer for Patients and Their Families. J Oncol Pract. 2015, 11(1): 12-18.

5. Howell D, Oliver TK, Keller-Olaman S, Davidson JR, Garland S, Samuels C, et al. Sleep disturbance in adults with cancer: a systematic review of evidence for best practices in assessment and management for clinical practice. Ann Oncol. 2014; 25(4): 791-800.

6. Merz EL, Tomfohr-Madsen L. Sleep Disruption in Pediatric Cancer Survivors: Conceptual Framework and Opportunities for Clinical Assessment and Behavioral Treatment. Am J Lifestyle Med. 2016; 12(4): 311-323.

7. Mulrooney DA, Ness KK, Neglia JP, Whitton JA, Green DM, Zeltzer LK, et al. Fatigue and sleep disturbance in adult survivors of childhood cancer: a report from the childhood cancer survivor study (CCSS). Sleep. 2008; 31(2): 271-281.

8. Miaskowski C, Mastick J, Paul SM, Abrams G, Cheung S, Sabes JH, et al. Impact of chemotherapyinduced neurotoxicities on adult cancer survivors' symptom burden and quality of life. J Cancer Surviv. 2018; 12(2): 234-245.

9. Cheung YT, Lim SR, Ho HK, Chan A. Cytokines as mediators of chemotherapy-associated cognitive changes: current evidence, limitations and directions for future research. PloS one. 2013; 8(12): e81234.

10. Balachandran DD, Faiz S, Bashoura L, Manzullo E. Cancer-related fatigue and sleep disorders. Sleep Med Clin. 2013; 8(2): 229-234.

11. Chung OKJ, Li HCW, Chiu SY, Ho KYE, Lopez V. The impact of cancer and its treatment on physical activity levels and behavior in Hong Kong Chinese childhood cancer survivors. Cancer Nurs. 2014; 37(3), E43-E51. 
12. Brown LF, Kroenke K. Cancer-related fatigue and its associations with depression and anxiety: a systematic review. Psychosomatics. 2009; 50(5), 440-447.

13. Otte JL, Carpenter JS, Manchanda S, Rand KL, Skaar TC, Weaver M, et al. Systematic review of sleep disorders in cancer patients: can the prevalence of sleep disorders be ascertained?. Cancer Med. 2015; 4(2): 183-200.

14. Mollayeva T, Thurairajah P, Burton K, Mollayeva S, Shapiro CM, Colantonio A. The Pittsburgh sleep quality index as a screening tool for sleep dysfunction in clinical and non-clinical samples: A systematic review and meta-analysis. Sleep Med Rev. 2016; 25, 52-73.

15. Buysse DJ, Reynolds 3rd CF, Monk TH, Berman SR, Kupfer DJ. The Pittsburgh Sleep Quality Index: a new instrument for psychiatric practice and research. Psychiatry Res. 1989; 28(2): 193-213.

16. Beck SL, Schwartz AL, Towsley G, Dudley W, Barsevick A. Psychometric evaluation of the Pittsburgh Sleep Quality Index in cancer patients. J Pain Symptom Manage. 2004; 27(2): 140-148.

17. Fontes F, Gonçalves M, Maia S, Pereira S, Severo M, Lunet N. Reliability and validity of the Pittsburgh Sleep Quality Index in breast cancer patients. Support Care Cancer. 2017; 25(10): 3059-3066.

18. Tietze AL, Zernikow B, Michel E, Blankenburg M. Sleep disturbances in children, adolescents, and young adults with severe psychomotor impairment: impact on parental quality of life and sleep. Dev Med Child Neurol. 2014; 56(12): 1187-1193.

19. de la Vega R, Tomé-Pires C, Solé E, Racine M, Castarlenas E, Jensen MP, et al. The Pittsburgh Sleep Quality Index: Validity and factor structure in young people. Psychol Assess. 2015; 27(4): e22-e27

20. Raniti MB, Waloszek JM, Schwartz O, Allen NB, Trinder J. Factor structure and psychometric properties of the Pittsburgh Sleep Quality Index in community-based adolescents. Sleep. 2018; 41(6): 10.1093/sleep/zsy066.

21. Gelaye B, Lohsoonthorn V, Lertmeharit S, Pensuksan WC, Sanchez SE, Lemma S, et al. Construct validity and factor structure of the pittsburgh sleep quality index and epworth sleepiness scale in a multi-national study of African, South East Asian and South American college students. PloS one. 2014; 9(12): e116383.

22. Erwin AM, Bashore L. Subjective Sleep Measures in Children: Self-Report. Front Pediatr. 2017; 5: 22.

23. Nunnally JC. Pyschometric Theory. New York, NY: McGraw-Hill, 1978.

24. Carpenter JS, Elam JL, Ridner SH, Carney PH, Cherry GJ, Cucullu HL. Sleep, fatigue, and depressive symptoms in breast cancer survivors and matched healthy women experiencing hot flashes. Oncol Nurs Forum. 2014; 31(3): 591-5598.

25. Cole JC, Motivala SJ, Buysse DJ, Oxman MN, Levin MJ, Irwin MR. Validation of a 3-factor scoring model for the Pittsburgh sleep quality index in older adults. Sleep. 2006; 29(1): 112-116.

26. Magee CA, Caputi P, Iverson DC, Huang XF. An investigation of the dimensionality of the Pittsburgh Sleep Quality Index in Australian adults. Sleep Biol Rhythms. 2008; 6: 222-227.

27. Li HC, Chung OK, Ho KY. Center for Epidemiologic Studies Depression Scale for Children: psychometric testing of the Chinese version. J Adv Nurs. 2010; 66(11): 2582-2591. 
28. Ho KY, Li WHC, Lam KW, Chiu SY, Chan CFG. The Psychometric Properties of the Chinese Version of the Fatigue Scale for Children. Cancer Nurs. 2016; 39(5): 341-348.

29. Ho KY, Li WH, Lam KW, Chiu SY, Chan CFG. Psychometric properties of the Chinese version of the fatigue scale-adolescent. BMC cancer. 2015;15: 938.

30. Chan LF, Chow SM, Lo SK. Preliminary validation of the Chinese version of the Pediatric Quality of Life Inventory. Int J Rehabil Res. 2005; 28(3): 219-227.

31. Bracken BA, Barona A. State of the art procedures for translating, validating and using psychoeducational tests in cross-cultural assessment. Sch Psychol Int. 1991; 12(1-2): 119-132.

32. Polit DF, Beck CT. The content validity index: are you sure you know what's being reported? Critique and recommendations. Res Nurs Health. 2006; 29(5): 489-497.

33. Cohen J. Statistical power analysis for the behavioral sciences. 2nd ed. Hillsdale: Lawrence Erlbaum Associates Publishers; 1988.

34. Zeltzer LK, Recklitis C, Buchbinder D, Zebrack B, Casillas J, Tsao JCl, et al. Psychological status in childhood cancer survivors: a report from the Childhood Cancer Survivor Study. J Clin Oncol. 2009; 27(14), 2396-2404.

35. Fortier-Brochu E, Beaulieu-Bonneau S, Ivers H, Morin CM. Relations between sleep, fatigue, and health-related quality of life in individuals with insomnia. J Psychosom Res. 2010; 69(5): 475-483.

36. Manzar MD, BaHammam AS, Hameed UA, Spence DW, Pandi-Perumal SR, Moscovitch A, et al. Dimensionality of the Pittsburgh Sleep Quality Index: a systematic review. Health Qual Life Outcomes. 2018; 16(1): 89.

37. Zhu B, Xie M, Park CG, Kapella MC. Adaptation of the Pittsburgh Sleep Quality Index in Chinese adults with type 2 diabetes. J Chin Med Assoc. 2018; 81(3), 242-247.

38. Yung KP, Chung KF, Ho FY, Yeung WF, Ng THY. The Experience of Chronic Insomnia in Chinese Adults: A Study Using Focus Groups and Insomnia Experience Diaries. Behav Sleep Med. 2016; 14(4): 406428.

39. Liu Y, Zhang J, Lam SP, Yu MWM, Li SX, Zhou J, et al. Help-seeking behaviors for insomnia in Hong Kong Chinese: a community-based study. Sleep Med. 2016; 21: 106-113.

40. Sarris J, Byrne GJ. A systematic review of insomnia and complementary medicine. Sleep Med Rev. 2011; 15(2): 99-106.

41. Casement MD, Harrington KM, Miller MW, Resick PA. Associations between Pittsburgh Sleep Quality Index factors and health outcomes in women with posttraumatic stress disorder. Sleep Med. 2012; 13(6): 752-758.

42. Aloba OO, Adewuya AO, Ola BA, Mapayi BM. Validity of the Pittsburgh Sleep Quality Index (PSQI) among Nigerian university students. Sleep Med. 2007; 8(3): 266-270.

43. Backhaus J, Junghanns K, Broocks A, Riemann D, Hohagen F. Test-retest reliability and validity of the Pittsburgh Sleep Quality Index in primary insomnia. J Psychosom Res. 2002; 53(3): 737-740. 
44. Curcio G, Tempesta D, Scarlata S, Marzano C, Moroni F, Rossini PM, et al. Validity of the Italian version of the Pittsburgh Sleep Quality Index (PSQI). Neurol Sci. 2013; 34(4): 511-519.

45. Okajima I, Komada Y, Nomura T, Nakashima K, Inoue Y. Insomnia as a risk for depression: a longitudinal epidemiologic study on a Japanese rural cohort. J Clin Psychiatry. 2012; 73(3): 377383.

46. Sohn SI, Kim DH, Lee MY, Cho YW. The reliability and validity of the Korean version of the Pittsburgh Sleep Quality Index. Sleep Breath. 2012; 16(3), 803-12.

47. Osher D, Kendziora, K, Spier E, Garibaldi ML. School influences on child and youth development. In: Sloboda Z, Petras H, editors. Advances in prevention science: Vol. 1. Defining prevention science. New York: Springer; 2014. p151-169.

48. Zhou ES, Recklitis CJ. Insomnia in adult survivors of childhood cancer: a report from project REACH. Support Care Cancer. 2014. 22(11), 3061-3069.

49. Garland SN, Johnson JA, Savard J, Gehrman P, Perlis M, Carlson L, et al. Sleeping well with cancer: a systematic review of cognitive behavioral therapy for insomnia in cancer patients. Neuropsychiatr Dis Treat. 2014; 10: 1113-1124.

50. Waldron EA, Janke EA, Bechtel CF, Ramirez M, Cohen A. A systematic review of psychosocial interventions to improve cancer caregiver quality of life. 2013; 22(6): 1200-1207.

\section{Figures}




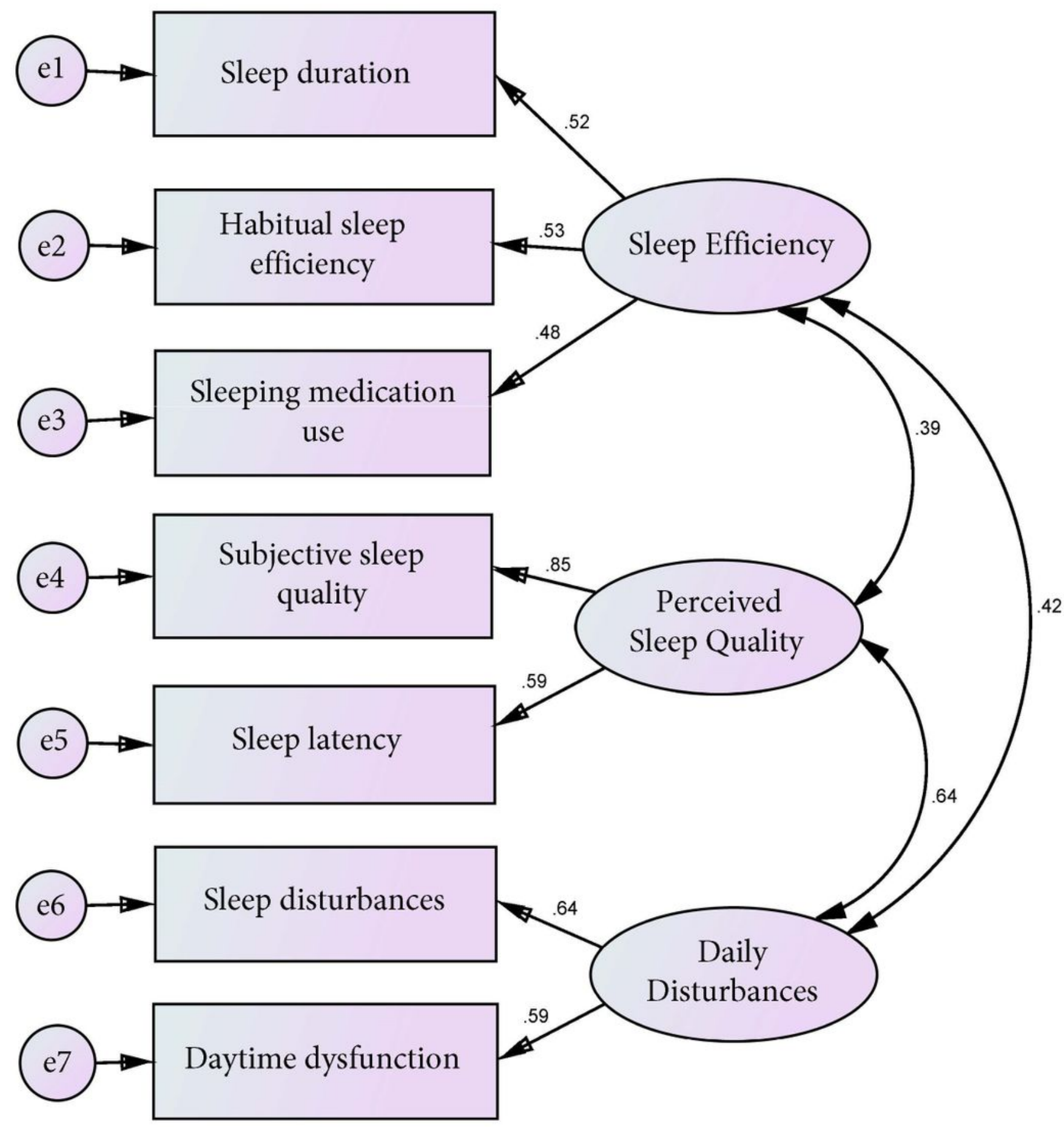

\section{Figure 1}

The estimated parameters of the Chinese version of the PSQI based on the three-factor model 


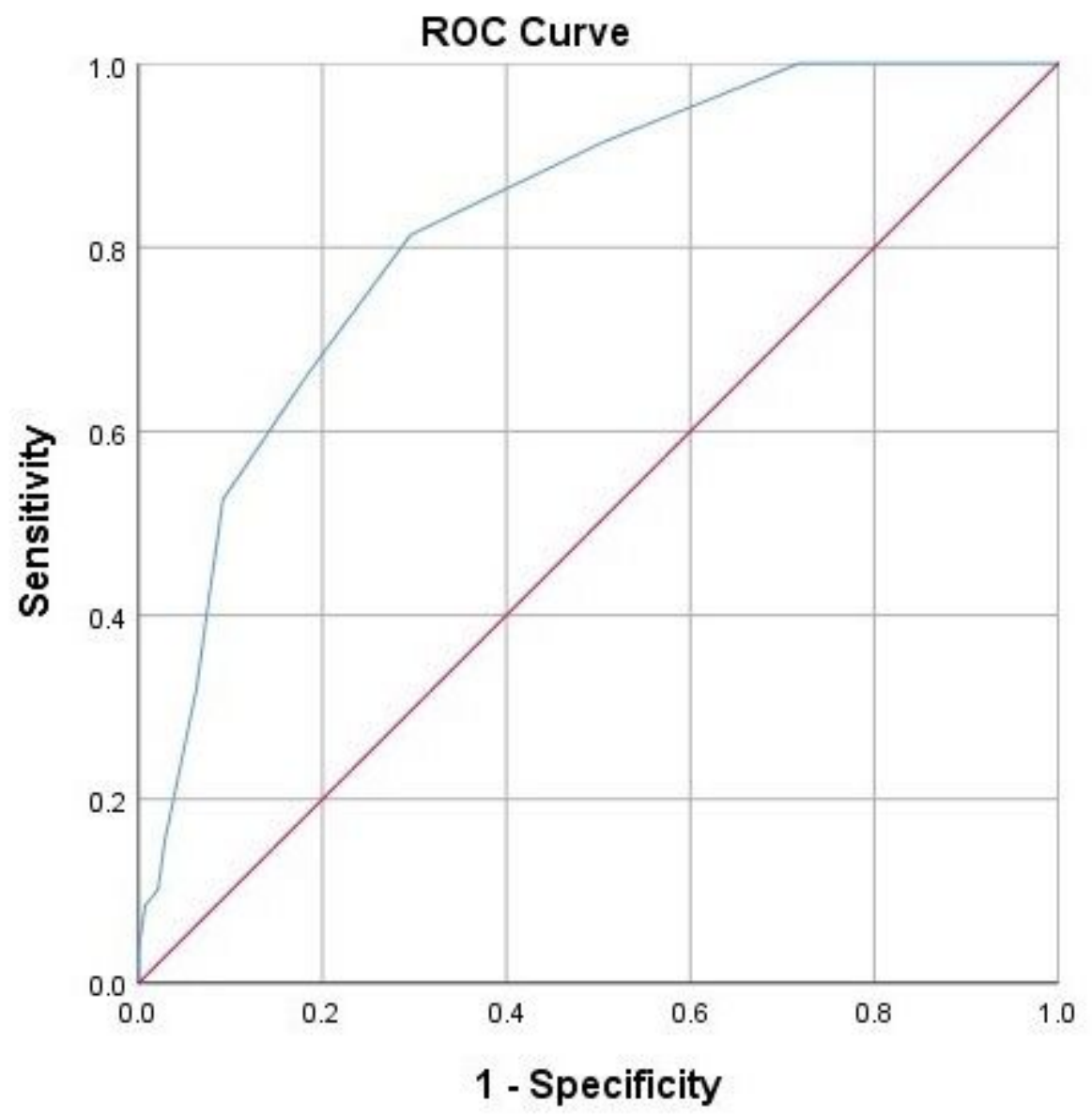

Diagonal segments are produced by ties.

Figure 2

The AUC of the Chinese version of the PSQI when compared with the DSM-5 diagnostic criteria for insomnia 